(multilayer state) and $280 \mathrm{~K}$ (monolayer state, desorption activation energy of $16.6 \mathrm{kcal} / \mathrm{mol}$ ). Hydrazine decomposed extensively into $\mathrm{NH}_{3}, \mathrm{~N}_{2}, \mathrm{H}$, and $\mathrm{N}$. B adatoms (electron-acceptor species) enhanced the bonding energy of hydrazine molecules (electron-donor species) on $\mathrm{Ru}(0001)$.

(2) Thermal desorption spectra from $\mathrm{N} / \mathrm{Ru}(0001)$ surfaces showed $\mathrm{N}_{2}$ evolution in a sharp peak at $\sim 510 \mathrm{~K}$ and in a broad feature between 600 and $800 \mathrm{~K}$.

(3) Boron-nitrogen adlayers with stoichiometry close to 1:1 were formed: (a) after coadsorption of $\mathrm{B}_{2} \mathrm{H}_{6}$ and $\mathrm{N}_{2} \mathrm{H}_{4}$ at $90 \mathrm{~K}$, and subsequent annealing to temperatures above $450 \mathrm{~K}$, and (b) by simultaneous dosing of $\mathrm{B}_{2} \mathrm{H}_{6}$ and $\mathrm{N}_{2} \mathrm{H}_{4}$ to $\mathrm{Ru}(0001)$ at $450 \mathrm{~K}$

(4) For films with $\theta_{N}<1 \mathrm{ML}$, adsorbed atomic oxygen reduced the stability of the boron-nitrogen adlayers by forming strong B-O bonds.

Acknowledgment. This work was supported by the Texas Advanced Research Program under Grant No. 160769.

Registry No. $\mathrm{B}_{2} \mathrm{H}_{6}, 19287-45-7 ; \mathrm{N}_{2} \mathrm{H}_{4}, 302-01-2 ; \mathrm{BN}, 10043-11-5$; $\mathrm{Ru}, 7440-18-8$

\title{
Fractal Surfaces of Gold and Platinum Electrodeposits. Dimensionality Determination by Scanning Tunneling Microscopy
}

\author{
J. M. Gómez-Rodriguez, A. M. Baró,* \\ Departamento de Fisica de la Materia Condensada, C-III, Universidad Autônoma de Madrid, 28049 Madrid, \\ Spain
}

L. Vázquez,

Instituto de Ciencias de Materiales, Sede B, CSIC, Departamento de Física Aplicada, C-XII, Universidad Autónoma de Madrid, 28049 Madrid, Spain

R. C. Salvarezza, ${ }^{\dagger}$ J. M. Vara,

Departamento de Quimica Física Aplicada, C-II, Universidad Autônoma de Madrid, 28049 Madrid, Spain

and A. J. Arvia

INIFTA, Universidad Nacional de La Plata, Sucursal 4, Casilla de Correo 16, 1900 La Plata, Argentina (Received: April 30, 1991; In Final Form: August 20, 1991)

\begin{abstract}
The structure of gold and platinum deposits grown on gold and platinum wire cathodes, respectively, by electroreduction of the corresponding oxide layers has been analyzed in terms of surface fractals by measuring the perimeter $L$ and area $A$ of intergranular voids. Perimeter and area were determined from scanning tunneling microscopy (STM) topographic images of the deposit surfaces. Fractal behavior, $L \propto A^{D / 2}$ with $D^{\prime}=1.5 \pm 0.1$, is found for both platinum and gold deposits. From these measurements, the fractal dimension of the surfaces was 2.5 . The $D^{\prime}$ value of the gold deposits decreases by aging in the electrolyte at $325 \mathrm{~K}$ as a consequence of surface diffusion processes.
\end{abstract}

\section{Introduction}

The kinetics of surface reactions on metals is extremely dependent on the topography of the reacting surfaces. Real systems exhibit complicated shapes with a high degree of irregularity or disorder. In these cases fractal geometry has been useful to characterize rough surfaces in very general terms. Metal films grown under nonequilibrium conditions exhibit complicated structures and rough surfaces. ${ }^{1-4}$ An important case is the process of metal electrodeposition either from aqueous solutions or from hydrous metal oxide layers in aqeous acid electrolytes. The first process can generate dendritic, dense radial and DLA patterns according to the growth conditions. ${ }^{2,3}$ The second process, although less studied, can generate thin metal films with columnar-like structures similar to those described for metal deposits grown from the vapor phase $e^{s}$ or thick metal film showing selfsimilar patterns. ${ }^{6}$ Fractal characterization of these structures is usually made in the micrometer to centimeter range so that little information is available on the fractal nature of the deposit surfaces at the nanometer level. In this paper we present an island type analysis ${ }^{7}$ based on determination of the perimeter $L$ and area $A$ of intergranular voids of metal electrodeposits measured by scanning tunneling microscopy (STM) as a method to investigate

\footnotetext{
${ }^{\dagger}$ Permanent address: INIFTA, La Plata, Argentina.
}

the fractal nature of the electrodeposit surface at the nanometer level and to estimate the corresponding fractal dimension $D$.

\section{Experimental Section}

Gold and platinum films (thickness $\cong 10^{-5}-10^{-4} \mathrm{~cm}$ ) were prepared in $0.5 \mathrm{M} \mathrm{H}_{2} \mathrm{SO}_{4}$ at $298 \mathrm{~K}$ electroreducing the corresponding oxide layers grown on polycrystalline gold and platinum wires (length $0.4 \mathrm{~cm}$; radius $0.05 \mathrm{~cm}$ ). The electrochemical procedure for the preparation of these metal deposits has been reported elsewhere. 5,8 The electrodeposition of the gold and platinum oxide layers was made by applying potential sweep rates of 0.02 and $0.005 \mathrm{~V} / \mathrm{s}$ for gold and platinum substrates, respectively. Under these conditions the growth rate of the electrodeposit

(1) Matsushita, M.; Sano, M.; Hayakagawa, Y.; Honyo, H.; Sawada, Y. Phys. Rev. Lett. 1984, 53, 286.

(2) Grier, D. G.; Kessler, D. A.; Sander, L. M. Phys. Rev. Lett. 1987, 59, 2315.

(3) Brady, R. M; Ball, R. C. Nature 1984, 309, 225.

(4) Witten, T. A.; Sander, L. M. Phys. Rev. Lett. 1981, 47, 1400

(5) Vaazquez, L.; Bartolomé, A.; Barô, A. M.; Alonso, C.; Salvarezza, R. C.; Arvia, A. J. Surf. Sci. 1989, 215, 171.

(6) Pajkossy, T. J. Electroanal Chem. 1991, 300, 1.

(7) Feder, J. Fractals; Plenum Press: New York, 1988.

(8) Chialvo, A. C.; Triaca, W. E.; Arvia, A. J. J. Electroanal. Chem. 1984, 71,303 . 

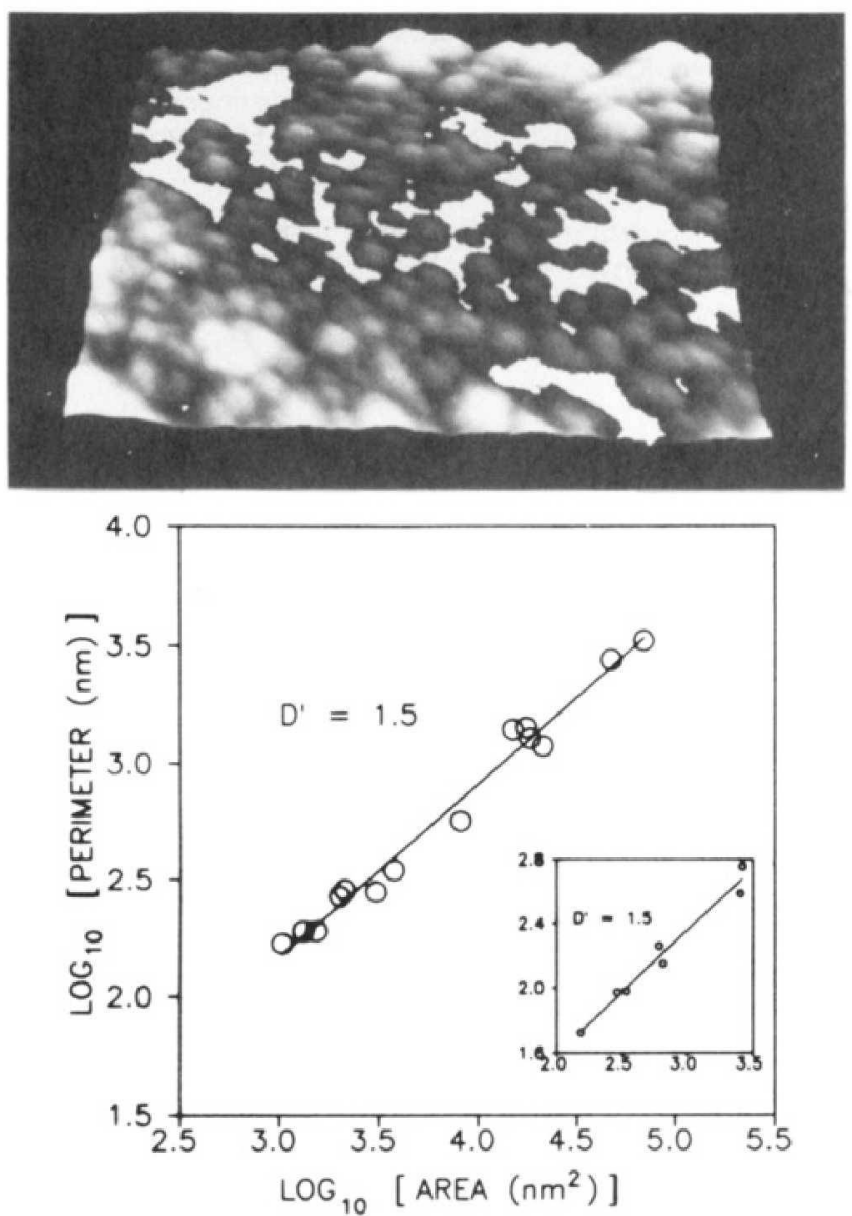

Figure 1. (a, top) $1400 \times 1000 \mathrm{~nm}^{2}$ 3D STM image of a nonaged gold electrodeposit with voids filled as lakes. (b, bottom) $\log L$ vs $\log A$ plot for the voids shown in (a). The yardstick is $\delta=5,6 \mathrm{~nm}$. The inset in this figure corresponds to the analysis of a smaller area $\left(460 \times 460 \mathrm{~nm}^{2}\right)$ of the same sample. The yardstick is $\delta=1.8 \mathrm{~nm}$.

is in the $10^{-6}-10^{-5} \mathrm{~cm} / \mathrm{s}$ range, which is consistent with the development of columnar structures with large surfaces. ${ }^{9}$ The concept of surface area for these samples is a difficult one due to the various accidents like cracks, pores, and indentations that a surface can have. We measured the area $S$ of the deposits through the O-electroadsorption charge density $\left(0.4 \mathrm{mC} \mathrm{cm}^{-2}\right) .^{5,10}$ The interesting point about the structure of these samples is the great value of $S$ obtained. For typical deposits with thickness close to $10^{-4} \mathrm{~cm}$ we obtain $S=8 \mathrm{~cm}^{2}, 80$ times greater than that of the starting metal wires.

Other types of deposits were prepared by aging the gold and platinum deposits during different times $t_{\mathrm{g}}$ at open circuit in the $0.5 \mathrm{H}_{2} \mathrm{SO}_{4}$ solution at $325 \mathrm{~K}$. Under these experimental conditions there is a marked decrease in $S$ for gold (i.e., from 8 to $1 \mathrm{~cm}^{2}$ for $t_{\mathrm{g}}=2 \times 10^{4} \mathrm{~s}$ ) whereas for platinum only a slight decrease in $S$ is observed (i.e., from 8 to $6 \mathrm{~cm}^{2}$ for $t_{\mathrm{g}}=6 \times 10^{4} \mathrm{~s}$ ).

Characterization of the surface of the electrodes before electrodeposition was also made by measuring the time dependence of the current $I$ of the $\mathrm{Fe}(\mathrm{CN})_{6}{ }^{4-} / \mathrm{Fe}(\mathrm{CN})_{6}{ }^{3-}$ reaction $^{6}$ under diffusion control. In this case we observed $I \propto t^{-n}$ with $n=0.5$ in the $5 \times 10^{-3}$ to $10 \mathrm{~s}$ time range. As $D=2 n+1$, the surface of the starting electrodes wires has $D=2$.

STM measurements were all made in the constant-current mode with bias voltages between 0.25 and $0.80 \mathrm{~V}$ and tunneling currents between 1 and $2 \mathrm{nA}$. The data were acquired with a fully automated workstation and stored as digitized images. A detailed description of the STM measurements are reported in ref 5.

(9) Srolowitz, D.; Mazor, A.; Bukiet, B. J. Vac. Sci. Technol. A 1988, 6, 2371

(10) Trassati, S.; Petrii, O. A. Real Surface Area Measurements in Electrochemistry; Comission on Electrochemistry, IUPAC: Oxford, in press.
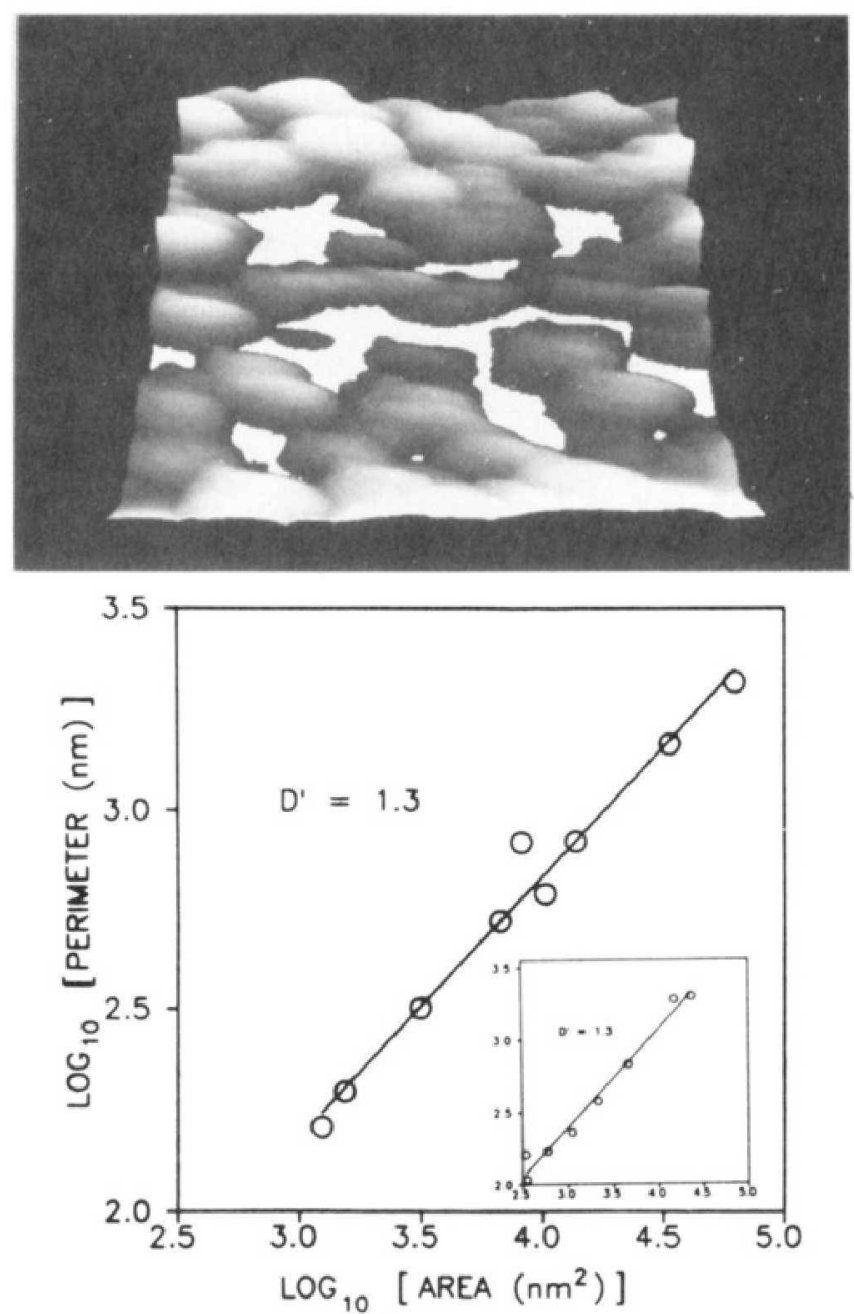

Figure 2. (a, top) $1100 \times 1100 \mathrm{~nm}^{2}$ 3D STM image of an aged gold electrodeposit $\left(t_{\mathrm{B}}=2 \times 10^{4} \mathrm{~s}\right.$ ) with voids filled as lakes. (b, bottom) $\log$ $L$ vs $\log A$ plot for the voids shown in (a). The yardstick is $\delta=4.5 \mathrm{~nm}$. The inset in this figure corresponds to the analysis of a smaller area (830 $\times 830 \mathrm{~nm}^{2}$ ) of the same sample. The yardstick is $\delta=3.2 \mathrm{~nm}$.

\section{Results and Discussion}

The fractal nature of the surfaces of thin metal films has been studied in recent years. ${ }^{11-13}$ Computer simulations reveal that the columnar units ${ }^{12}$ and the deposit surface ${ }^{13}$ are not self-similar but self-affine fractals, although certain controversy exists on this point. ${ }^{6,11}$ Thus, we propose a simple method to obtain the fractal dimension of surfaces in the nanometer range which applies to both self-similar and self-affine fractals. It is based on the fact that the intersection of a plane with a self-similar or a self-affine fractal surface generates self-similar lakes or islands. ${ }^{14}$ We analyze the fractal nature of the surface by ex situ STM imaging. Figure 1a is a STM 3D image of a nonaged gold electrodeposit. The time elapsed between formation and imaging was around 1 h. On the other hand, Figure 2 shows the 3D image of an aged gold deposit $\left(t_{\mathrm{g}}=2 \times 10^{4} \mathrm{~s}\right)$. In a way similar to that reported for the Earth coastlines ${ }^{14}$ we simulate the "filling with water" of the surface topography up to a given level. By using threshold detection and optimal connectivity checking algorithms, individual voids "filled with water" were isolated. Then, their perimeter $L$, defined as the number of water pixels on the digitized grid having non-water neighbors, and their area $A$, defined as the number of water pixels for a given void, were measured. The yardstick value $\delta$ of the digitized grid is given by the total area scanned and the

(11) Yehoda, J. E.; Messier, R. Applic. Surface Sci. 1986, 22/23, 590.

(12) Meakin, P. J.Phys. A 1987, 20, L1113.

(13) Family, A.; Vicsek, T. J. Phys. 1985, 18, L75.

(14) Mandelbrot, B. B. The Fractal Geometry of Nature; Freeman: San Francisco, 1977. 

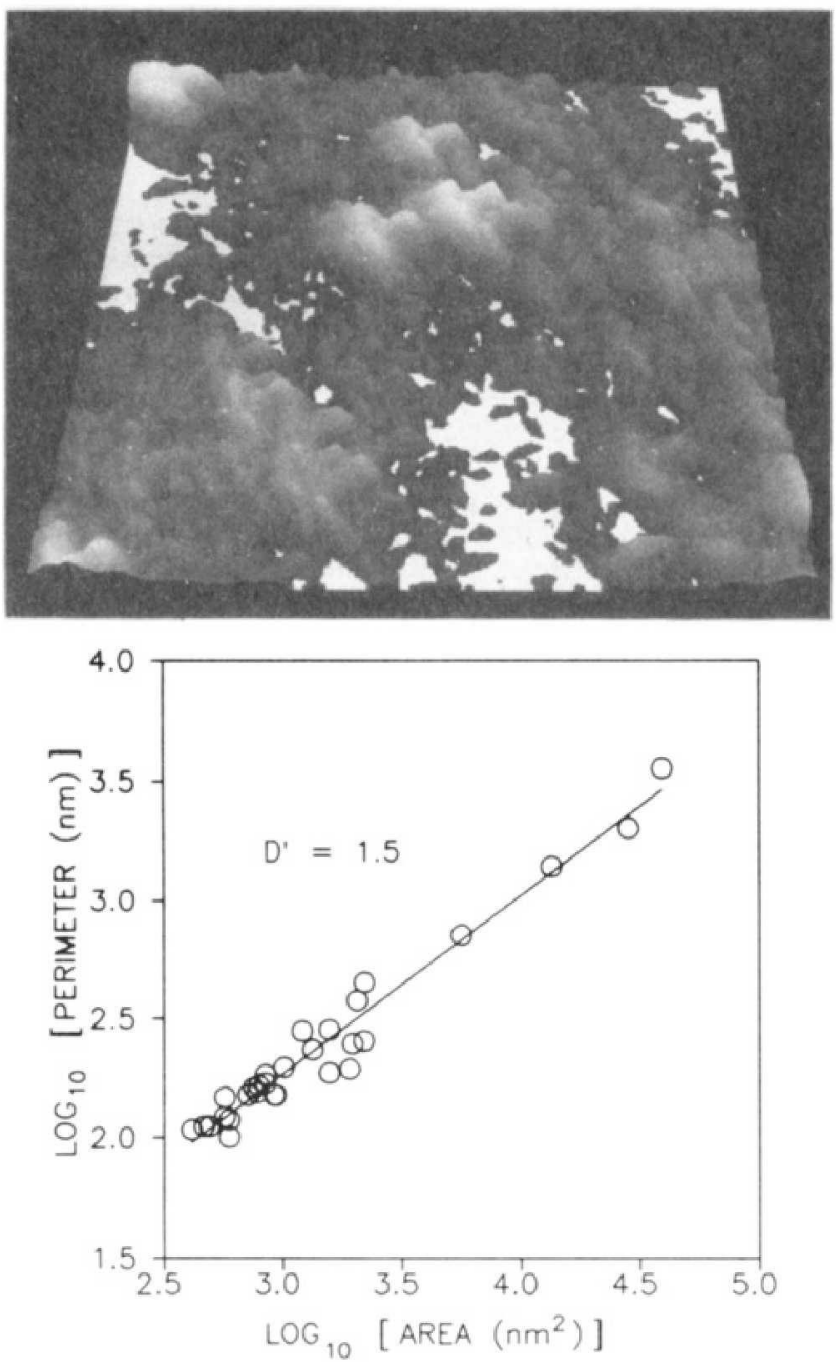

Figure 3. (a, top) $930 \times 890 \mathrm{~nm}^{2}$ 3D STM image of a nonaged platinum electrodeposit with voids filled as lakes. (b, bottom) $\log L$ vs $\log A$ plot for the voids shown in (a). The yardstick is $\delta=3.6 \mathrm{~nm}$.

number of pixels in the image (typically $256 \times 256$ ). An initial point of concern is the minimum lake size that one should consider, since the measurement of the length and area of a lake in a digitized grid requires $\delta$ to be small enough so as to account for the smaller features. Kaye ${ }^{15}$ suggests that a rugged perimeter has to be measured with a yardstick smaller than 0.3 times the projected total length. We have been analyzing several images in order to determine empirically the more suitable choice. As our yardstick is fixed for each image (i.e., the yardstick is equal to one pixel length), we have considered only lakes with an area greater than $30 \delta^{2}$, which is a more restricted condition than Kaye's.

$L$ and $A$ for similar shaped objects are related by ${ }^{7}$

$$
L=\alpha D^{\prime} A^{D / 2}
$$

where $\alpha$ is a constant and $D^{\prime}$ is the fractal dimension of the void perimeter. From the rule of thumb $b^{7,14}$ it follows

$$
D^{\prime}=D-1
$$

where $D$ is the fractal dimension of the deposit surface.

The $\log L$ vs $\log A$ plot, covering two decades in area scale, yields a straight line with $D^{\prime}=1.5 \pm 0.06$ and $D^{\prime}=1.3 \pm 0.06$ for nonaged and aged gold electrodeposits, respectively (Figures $1 \mathrm{~b}$ and $2 \mathrm{~b}$ ). The yardstick value in Figure $1 \mathrm{~b}$ was $5.6 \mathrm{~nm}$, while in Figure 2b it was $4.5 \mathrm{~nm}$. We show as insets in Figures $1 \mathrm{~b}$ and $2 \mathrm{~b}$ the plots obtained from different STM measurements on smaller areas of the electrodeposits. Though the yardstick were

(15) Kaye, B. H. In The Fractal Approach to Heterogeneous Chemistry; Avnir, D., Ed.; Wiley: New York, 1989; p 58.
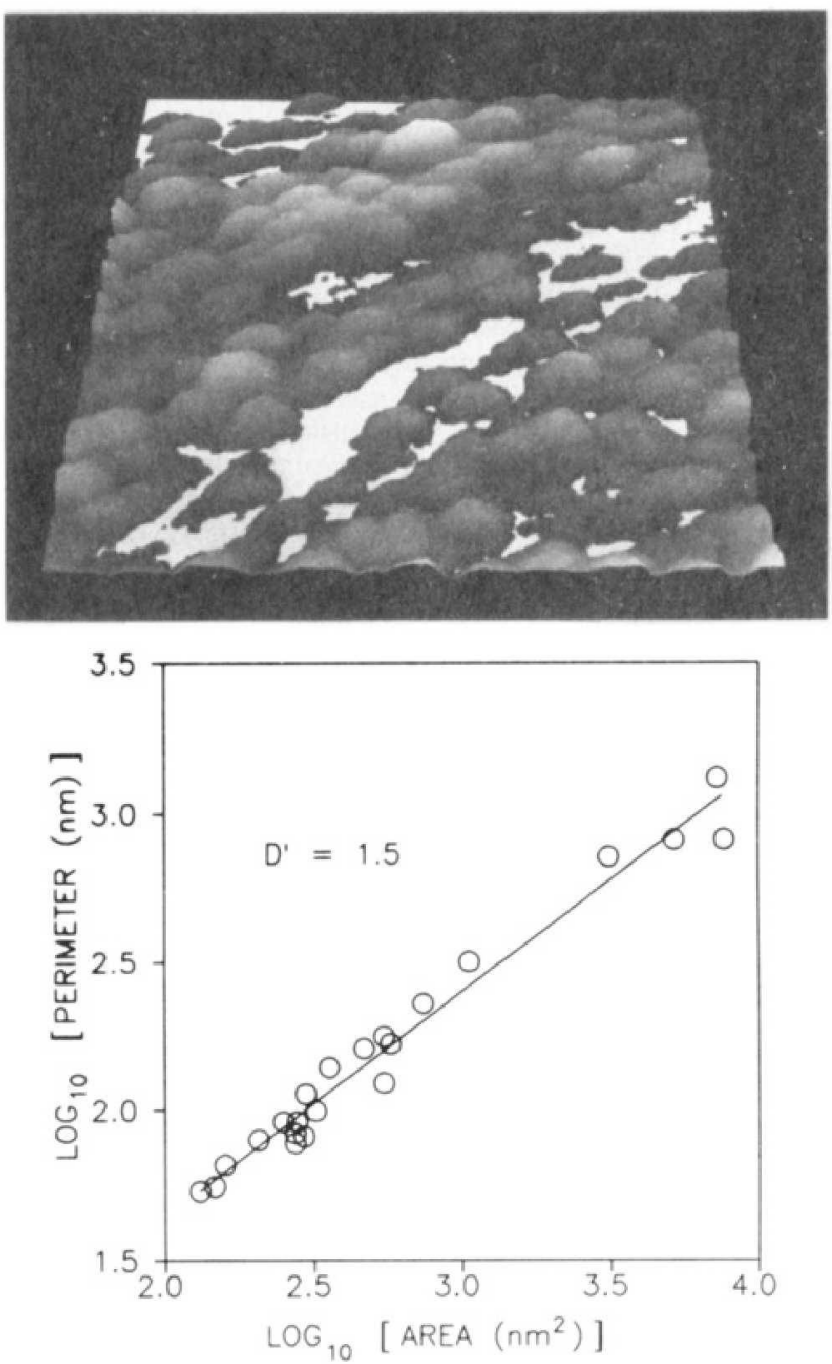

Figure 4. (a, top) $510 \times 490 \mathrm{~nm}^{2}$ 3D STM image of an aged platinum electrodeposit $\left(t_{g}=4 \times 10^{4} \mathrm{~s}\right)$ with voids filled as lakes. (b, bottom) $\log$ $L$ vs $\log A$ plot for the voids shown in (a). The yardstick is $\delta=2.0 \mathrm{~nm}$.

smaller, the $D^{\prime}$ values remained the same: $D^{\prime}=1.5 \pm 0.06$ and $D^{\prime}=1.3 \pm 0.06$ for the nonaged and aged gold electrodeposits, respectively.

The same "filling with water" procedure was performed on nonaged and aged $\left(t_{\mathrm{g}}=4 \times 10^{4} \mathrm{~s}\right)$ platinum electrodeposits. Their 3D STM images (Figures 3a and 4a) looked similar to those of the gold surfaces (Figures $1 \mathrm{a}$ and $2 \mathrm{a}$ ). From the generated void patterns the $\log L$ vs $\log A$ plots were obtained (Figures $3 \mathrm{~b}$ and $4 \mathrm{~b})$. However, in this case, one obtains $D^{\prime}=1.5 \pm 0.06$ for both types of deposits.

A limitation of the STM method is related to the fact that the tip itself limits the roughness resolution as the STM images are the convolution of the surface with the tip shape. A quantitative estimation of the tip shape effect requires the knowledge of the real tip geometry, ${ }^{16}$ which is rather difficult in practice. Thus, we used an experimental approach to solve this problem analyzing several sample sets $\left(2 \times 10^{-5} \mathrm{~cm}<\right.$ film thickness $\left.<2 \times 10^{-4} \mathrm{~cm}\right)$ using different STM probing tips. We were not able to detect any marked tip influence on the $D$ values, which remained practically constant within \pm 0.1 error. A similar observation was already reported for metal fracture surfaces. ${ }^{16}$ The fact that the tip geometry has not influenced our results could be explained considering that the smallest lakes which should be mostly affected by the tip shape are discarded due to the more restrictive condition imposed by the perimeter/area measurements. This limitation was also analyzed by using several water-filling heights in a $\pm 5-\mathrm{nm}$ range, yielding again a $D^{\prime}$ nearly independent (within \pm 0.1 ) on 
the depth at which the surface was studied. Thus, in our experimental system, we cannot determine $D$ with an error less than 0.1 .

From eq $2, D$ values of $2.5 \pm 0.1$ and $2.3 \pm 0.1$ result for the nonaged and aged gold surfaces, respectively, in agreement with the smoothing of the deposit surface as $t_{\mathrm{g}}$ increases (Figures $1 \mathrm{a}$ and $2 \mathrm{a}$ ). These values indicate a marked reconstruction of the gold surface in order to decrease the surface area energy of the system. On the other hand, the $D$ value for nonaged and aged platinum surfaces is $2.5 \pm 0.1$, showing that platinum deposits are more stable than gold deposits under similar experimental conditions. This conclusion is consistent with the slower mobility of the platinum surface (surface diffusion coefficient, $D_{\mathrm{s}, \mathrm{Pt}} \cong 1$ $\left.\times 10^{-18} \mathrm{~cm}^{2} / \mathrm{s}\right)^{17}$ in relation to the surface mobility of gold $\left(D_{\mathrm{s}, \mathrm{Au}}\right.$ $\left.\cong 1 \times 10^{-13} \mathrm{~cm}^{2} / \mathrm{s}\right)^{17}$ in this electrolyte and temperature. Similar results were recently reported on the surface diffusion of gold and platinum in air at room temperature. ${ }^{18}$ The effect of the aging process on the $D$ values was verified by measuring the time dependence of the diffusional current of the $\mathrm{Fe}(\mathrm{CN})_{6}{ }^{4-} / \mathrm{Fe}(\mathrm{CN})_{6}{ }^{3-}$ reaction. ${ }^{6}$ We obtained $D=2.5 \pm 0.1$ and $D=2.3 \pm 0.1$ for recently prepared and aged gold electrodeposits in the $5 \times 10^{-3}$ and $0.1 \mathrm{~s}$ time range, ${ }^{19}$ respectively, in good agreement with the STM results. On the other hand, the value of $D=2.5 \pm 0.1$ obtained for our platinum electrodeposits by the STM method is also in good agreement with $D=2.46$ reported for a thicker platinum deposit exhibiting self-similar patterns obtained by the same electrochemical procedure. ${ }^{6}$

Finally, it should be noted that information concerning the growth mechanism of the deposits should be derived from the analysis of the $D$ value of nonaged samples $(D \cong 2.5)$. For diffusion-limited deposition with DLA patterns one expects $D=$ $2.5^{3}$ whereas for a simple ballistic deposition $D^{\prime}$ gives $1.67,{ }^{20}$ both

(17) Alonso, C.; Salvarezza, R. C.; Vara, J. M.; Arvia, A. J.; Vâzquez, L.; Bartolomé, A.; Barô, A. M. J. Electrochem. Soc. 1990, 137, 2161.

(18) Sommerfeld, D. A.; Cambron, R.; Beebe, T., Jr. J. Phys. Chem. 1990 94,8926

(19) Ocon, P.; Herrasti, P.; Vázquez, L.; Salvarezza, R. C.; Vara, J. M.; Arvia, A. J. J. Electroanal. Chem., in press. in three dimensions. Our experimental values of $D$ are closer to that expected for DLA model. Computer simulations and experimental work are in progress in our laboratory to verify this point.

\section{Conclusions}

(1) We have used STM images to measure the fractal dimension of the surface of thin gold and platinum electrodeposits by using perimeter/area relationships. This island type analysis, which applies to both self-similar and self-affine fractals, is based on the fact that STM is able to give real-space three-dimensional images of the surface. Also the good lateral resolution of STM allows us to reach the nanometer range, and the data show that the fractal nature of the surface reaches this small unit.

(2) For freshly prepared gold and platinum electrodeposits, a $D$ value equal to $2.5 \pm 0.1$ was obtained. The aging of the gold films in the electrolyte at $325 \mathrm{~K}$ produced a decrease in the $D$ value from $2.5 \pm 0.1$ to $2.3 \pm 0.1$, whereas no change in $D$ was detected for aged platinum films. The correlation of this with surface diffusivities suggests that diffusion of atoms on surfaces can cause changes over time of fractal dimension, ${ }^{21}$ and different values of this dimension should be obtained by changing the metal, electrolyte composition, and temperature.

Acknowledgment. A fellowship from the Comision Interministerial de Ciencia y Tecnologia (Spain) and Consejo Nacional de Investigaciones Cientificas y Técnicas (Argentina) to R.C.S. is gratefully acknowledged. Financial support was obtained from the CICYT through Contract No. MAT89-0204. We acknowledge the critical reading of the manuscript by F. Guinea. We also thank J. Gómez-Herrero for valuable discussions.

Registry No. Au, 7440-57-5; $\mathrm{Pt}, 7440-06-4 ; \mathrm{H}_{2} \mathrm{SO}_{4}, 7664-93-9$; $\mathrm{Fe}-$ $(\mathrm{CN})_{6}{ }^{3-}, 13408-62-3 ; \mathrm{Fe}(\mathrm{CN})_{6}{ }^{4-}, 13408-63-4$; gold oxide, 39403-39-9; platinum oxide, $11129-89-8$

(20) Vicsek, T. Fractal Growth Phenomena; World Scientific: Singapore, New Jersey, London, 1989.

(21) Hepel, T. J. Electrochem. Soc. 1987, 134, 2687.

\title{
Catalytic and Spectroscoplc Studles of the Water Gas Shift Reaction over RuNa-X and RuH-X Zeolltes
}

\author{
Guan-dao Lei and Larry Kevan* \\ Department of Chemistry, University of Houston, Houston, Texas 77204-5641 (Received: May 7, 1991)
}

\begin{abstract}
The low-temperature water gas shift reaction has been studied over $\mathrm{Ru}\left(\mathrm{NH}_{3}\right)_{6} \mathrm{Cl}_{3}$ exchanged into the $\mathrm{H}-\mathrm{X}$ and $\mathrm{Na}-\mathrm{X}$ zeolites. The RuNa-X and RuH-X zeolite catalysts are investigated under water gas shift reaction conditions by electron spin resonance, electron spin echo modulation, and infrared and diffuse reflectance spectroscopies. A Ru(II)-dicarbonyl complex is found to be the catalytically active species for the reaction, and the evidence suggests that it is located at a four-ring site in the $\alpha$-cage of the X zeolite. In the RuH-X zeolite, an inactive $\mathrm{Ru}$ species is formed inside the $\beta$-cage, which is responsible for its lower activity compared to the RuNa-X zeolite.
\end{abstract}

\section{Introduction}

The conversion of carbon monoxide and water to produce carbon dioxide and hydrogen as in eq 1 is known as the water gas

$$
\mathrm{CO}+\mathrm{H}_{2} \mathrm{O} \rightarrow \mathrm{CO}_{2}+\mathrm{H}_{2}
$$

shift (WGS) reaction. Its industrial importance derives from its role in the production of ammonia gas, ${ }^{1}$ which requires high-purity hydrogen, and from its ability to increase the $\mathrm{H}_{2}: \mathrm{CO}$ ratio in

(1) Newsome, D. S. Catal. Rev.-Sci. Eng. 1980, 21, 275. gaseous feedstock for methanation and Fisher-Tropsch synthesis. ${ }^{2}$ Commercially, the WGS reaction is carried out over iron-based and copper-based catalysts, which operate at temperatures of $300-400$ and $200-250^{\circ} \mathrm{C}$ and are called high-temperature shift catalysts and low-temperature shift catalysts, respectively. Attempts have been made to use homogeneous catalysts for the WGS reaction such as rhodium carbonyl iodide and ruthenium carbonyl in alkaline solution. ${ }^{3,4}$ Ruthenium supported on faujasite-type

(2) Storch, H. H.; Golumbic, N.; Anderson, R. B. The Fisher-Tropsch and Related System; Wiley: New York, 1951. 